\title{
General mechanism of JQ1 in inhibiting various types of cancer
}

\author{
GUOJUAN JIANG $^{1 *}$, WANGLONG DENG ${ }^{1 *}$, YANG LIU $^{1}$ and CHENGDE WANG ${ }^{2}$ \\ ${ }^{1}$ State Key Laboratory of Medical Genomics, Shanghai Institute of Hematology, Rui Jin Hospital Affiliated to \\ School of Medicine, Shanghai Jiao Tong University, Shanghai $200025 ;{ }^{2}$ Department of Neurosurgery, \\ The First Affiliated Hospital of Wenzhou Medical University, Wenzhou, Zhejiang 325000, P.R. China
}

Received March 29, 2019; Accepted October 4, 2019

DOI: $10.3892 / \mathrm{mmr} .2020 .10927$

\begin{abstract}
Bromodomain-containing 4 (BRD4) is a histone modification reader and transcriptional regulator that has been reported to interact with acetylated lysine histone motifs transcription factors (TFs), transcription co-activators and RNA polymerase II. The selective small molecule inhibitor JQ1, which binds competitively to bromodomains, has been reported to exhibit anti-proliferative effects in various types of cancer. Previous studies on the mechanism of action of JQ1 mostly focused on a specific tumor type or disease; however, the general mechanism through which JQ1 affects various tumors remains to be determined. In the present study, chromatin immunoprecipitation sequencing data for BRD4 and its expression profiles in six cancer cell lines were integrated and analyzed systematically. The results indicated that BRD4 binds to enhancers with histone $\mathrm{H} 3$ acetylated at lysine $27(\mathrm{H} 3 \mathrm{~K} 27 \mathrm{Ac})$ and mediator complex subunit 1 in a cell type-specific manner, as well as binds to promoter regions with the oncogenic TFs MYC and E2F1 in a cell type-common manner. The cell type-common sites across the six cell types investigated were found to be functionally important for tumorigenesis, whereas the cell type-specific sites were functionally enriched with the cell identity, all of which were sensitive to JQ1 treatment. Furthermore, a core set of JQ1-regulated BRD4 binding genes were obtained, which were significantly inhibited by JQ1 in various cancer cell lines and contributed to hallmarks of cancer. These results implied
\end{abstract}

Correspondence to: Dr Chengde Wang, Department of Neurosurgery, The First Affiliated Hospital of Wenzhou Medical University, 2 Fu Xue Lane, Wenzhou, Zhejiang 325000, P.R. China E-mail: yihe723@126.com

Dr Guojuan Jiang, State Key Laboratory of Medical Genomics, Shanghai Institute of Hematology, Rui Jin Hospital Affiliated to School of Medicine, Shanghai Jiao Tong University, 227 Chongqing South Road, Shanghai 200025, P.R. China

E-mail: guojuanjiang2018@126.com

*Contributed equally

Key words: small molecular compound, bromodomain-containing 4 inhibitor, JQ1 a common mechanism underlying the therapeutic effects of JQ1 and suggested its potential suitability as an anti-cancer drug targeting BRD4-mediated transcriptional regulation.

\section{Introduction}

Bromodomain (BD) and BD extra-terminal domain (BET) protein family members, including BD-containing 2 (BRD2), BRD3, BRD4 and BD testis-associated (BRDT), specifically recognize acetylated lysine acid on histones mainly through two conserved N-terminal regions (BD1 and BD2) $(1,2)$. BET proteins bind to the acetylation site on the chromosome and recruit other transcriptional regulatory complexes to regulate gene expression. Their ability to bind to a single acetylation site on histones is generally weak, whereas their ability to bind to multiple acetylation sites is strong (3).

BRD4 is the most well-studied and important functional member of the BET protein family. Its binding characteristics and vital functions in transcriptional regulation have been extensively reported. BRD4 interacts with multiple protein complexes in the active promoter and enhancer regions, including Mediator, positive transcription elongation factor $b$ (P-TEFb) and jumonji domain containing 6 (Jmjd6), and serves multiple roles in transcriptional regulation $(4,5)$. BRD4 was first discovered during the isolation and purification of human Mediator complexes (6). Subsequent studies have indicated that BRD4 and Mediator interact in multiple cell types and have similar binding distributions across the chromosome. This co-localization helps them bind more stably to DNA (6). The Mediator complex is able to link the transcription factor (TF) activation effect to RNA polymerase II (RNAPII), while the interaction of BRD4 with Mediator suggests that it serves an important role in TF-mediated transcriptional activation. During RNA transcription, the transcriptional initiator RNAPII binds to the transcriptional arrest-associated factors DRB sensitivity-inducing factor (DSIF) and negative elongation factor (NELF), halting transcription at a position located 20-60 nt downstream of the transcription initiation site. P-TEFb is a transcription elongation factor that phosphorylates DSIF and NELF. Once recruited to the promoter region, $\mathrm{P}-\mathrm{TEFb}$ promotes RNAPII elongation by phosphorylating DSIF and NELF (7). Previous studies have indicated that BRD4 recognizes the acetylation site of the activated promoter region, recruits P-TEFb to the vicinity of the gene promoter and links the active chromatin 
region in the acetylated state to RNAPII for extension (4). It has also been reported that BRD4 forms a protein complex with the histone demethylase Jmjd6 in the distal enhancer region to promote RNAPII-mediated elongation (8). These distal enhancers are known as anti-pause enhancers, which also suggests that the complexes formed by BRD4, Jmjd6 and $\mathrm{P}-\mathrm{TEFb}$ bind to chromatin through long-distance interactions, as they are close to the distal and deep promoters of the gene in three-dimensional space, thus regulating transcriptional activation and elongation of genes (8-10). The majority of studies investigating BRD4 are limited to specific cell types and are based on in vitro biochemical experiments. Therefore, at the genome-wide level, the integration of multi-level chromatin immunoprecipitation-sequencing (ChIP-seq) data from multiple cell lines is of great significance for comprehensive and in-depth analyses of the binding characteristics of BRD4.

The BET inhibitors JQ1 and I-BET were developed by two independent research groups and reported in $2010(11,12)$. The inhibitors compete with acetylation sites for histone binding to the BD of the BET protein, making it impossible for the BET protein to interact with the histones and resulting in dissociation of the BET protein from the chromatin. The discovery of BET inhibitors has made BRD4 a potential target for the treatment of a variety of diseases, particularly cancer. The BRD4-NUT (nuclear protein in testis) fusion protein is a driver TF for midline cancer. JQ1 may cause detachment of BRD4-NUT from the chromatin, which promotes terminal differentiation and apoptosis of cancer cells. Recently, a large number of studies have indicated that BET inhibitors exhibit good therapeutic effects on hematopoietic malignancies, including acute myeloid leukemia (AML) $(13,14)$, multiple myeloma (MM) (15), Burkitt's lymphoma (BL) (16), diffuse large B-cell lymphoma (DLBCL) (17) and T-cell acute lymphoblastic leukemia (T-ALL) (18). In addition to hematological tumors, BET inhibitors also have good effects on a range of solid tumor types. It has been reported that neuroblastoma $(19,20)$ and medulloblastoma (20) are triggered by amplification of N-MYC and MYC, respectively, which are sensitive to JQ1 treatment, and their proliferation is negatively influenced by inhibition of N-MYC or MYC by JQ1. In a human lung adenocarcinoma cell line, JQ1 inhibited proliferation by reducing the expression of the proto-oncogene FOS-like 1, AP-1 TF subunit (FOSL1) and its downstream target genes (21). In addition, in activated endothelial cells and macrophages, JQ1 eliminated the inflammatory response mediated by super-enhancers, thereby exerting an anti-inflammatory effect. The majority of previous studies have linked the anti-cancer effect of BET inhibitors to their inhibition of important proto-oncogenes, including MYC and its downstream target genes. Subsequent studies have reported that a similar therapeutic effect to that of BET inhibitors may also be achieved by inhibiting other proto-oncogenes, including B-cell lymphoma 2 (BCL2) and E2F1, or key TFs, including FOSL1 and androgen receptor (AR) (22). Notably, overexpression of MYC or BCL2 in BET inhibitor-treated cancer cells has been reported to only partially attenuate the anti-cancer effect of the BET inhibitors $(15,16,19)$. This finding may indicate that the target of the BET inhibitor is not a single gene, but more likely includes multiple genes involved in the regulation of cancer. Based on this, it may be speculated that BET inhibitors have significant therapeutic effects on a variety of cancer types of different origins and pathogeneses, possibly by inhibiting certain key proto-oncogenes or a core gene set associated with cancer development to thereby interfere with proliferation, induce cell cycle arrest and trigger apoptosis.

BRD4 is an important member of the RNAPII transcriptional complex and is a co-activator of transcriptional regulation. However, the mechanisms of BET inhibitors exhibit distinct heterogeneity among different cell types. A super-enhancer is a small group of classic enhancer regions with super-enrichment of the co-activator, mediator complex subunit 1 (MED1) (23-25). The genes associated with super-enhancers are mostly cell-specific genes that determine cell identity. BRD4 is highly enriched in these super-enhancer regions and actively regulates the expression of this small group of important genes (26). BET inhibitors result in the dissociation of BRD4 from these regions, thereby selectively inhibiting super-enhancer-associated genes. However, a large number of studies have reported that BRD4 directly interacts with multiple TFs through $\mathrm{BD}$ recognition of acetylated lysines in TFs, including nuclear factor- $\kappa \mathrm{B}$, GATA-binding protein 1 and AR (22), or by undetermined means, which may involve P53, YY1 TF and MYC/MYC-associated factor $\mathrm{X}$ (MAX) (27). BET inhibitors not only directly inhibit the expression of key TFs, but also disrupt the binding of BRD4 to TFs in order to inhibit the activation of downstream target genes. The binding characteristics of BRD4 and the mechanism of action of BET inhibitors have been reported in specific cell types; however, the integration of multi-omics data from multiple cell lines to investigate the general mechanism in various types of cancer has not been previously performed. Such an analysis will provide a more unified theoretical basis for cancer treatment.

In the present study, the binding characteristics of BRD4 were systematically evaluated in multiple cell lines by integrating a set of ChIP-seq datasets for BRD4 and its associated factors in six different cancer cell types, as well as the expression profiles of the cells following JQ1 treatment (data provided in Tables SI and SII). The cell lines investigated in the current study included the human small cell lung cancer (SCLC) cell line H2171, the glioblastoma multiforme cell line U-87 MG, the MM cell line MM.1S, the DLBCL cell line cLy1, the T-ALL cell line KOPT-K1 and the tumor necrosis factor (TNF)- $\alpha$-activated human endothelial cell line HUVEC-C (28). The results of the current study revealed the general mechanism of BET inhibitors in the treatment of cancer. Notably, a core gene set that is regulated by JQ1 and is important for the progression of various cancer types was identified, which provides potential targets for cancer therapy, as well as a broader and more uniform perspective for understanding the mechanism underlying the action of BET inhibitors.

\section{Materials and methods}

Data integration. For comprehensive analysis of BRD4 binding across the genome, ChIP-seq data were collected for multiple factors, including BRD4, H3K27Ac, RNAPII, MED1, MYC, MAX, E2F1 and histone H3 trimethylated at lysine 4 (H3K4me3), in six tumor cell lines (namely H2171, U-87 MG, 
MM.1S, Ly1, KOPT-K1 and HUVEC-C). H2171 (ATCC ${ }^{\circledR}$ CRL-5929 ${ }^{\mathrm{TM}}$ ) used in the present study was a human SCLC cell line. The U-87 MG (ATCC ${ }^{\circledR}$ HTB-14 ${ }^{\mathrm{TM}}$ ) cell line was also used, which is most probably a glioblastoma cell line of unknown origin. The MM cell line MM.1S (ATCC ${ }^{\circledR}$ CRL-2974 ${ }^{\mathrm{TM}}$ ) was also examined, as well as the DLBCL cell line Lyl, which has previously been used in the study of Chapuy et al (17). In addition, the T-ALL cell line KOPT-K1 was used, which was kindly gifted by Dr A.T. Look from Dana-Farber Cancer Institute (Boston, MA, USA) (16). The TNF- $\alpha$-activated human endothelial cell line HUVEC-C (ATCC ${ }^{\circledR}$ CRL-1730 ${ }^{\mathrm{TM}}$ ) was also used in the current study, which has previously been investigated in the study of Brown et al (28).

Raw ChIP-seq data were downloaded from the Sequence Read Archive (SRA; http://www.ncbi.nlm.nih.gov/sra) of the National Center for Biotechnology Information (NCBI). According to the unified processing analysis pipeline, the detailed information and analysis results are provided in Table SI. For analysis of the effects of JQ1 on gene expression in the corresponding cell lines, the gene expression profiles of multiple cell lines prior to and following JQ1 treatment were also integrated. Raw data for all cell lines were downloaded from the NCBI Gene Expression Omnibus (GEO; http://www. ncbi.nlm.nih.gov/geo/) database. The accession numbers and microarray data are provided in Table SII. The collected gene expression profiles were mainly divided into three categories, as follows: Gene expression profiles treated with JQ1 matched with the corresponding ChIP-seq data served as the training set; Expression profiles treated with JQ1 without corresponding ChIP-seq data served as the test set; and a class of normal gene expression data without JQ1 treatment for all the six cell lines under the same platform served as the expression set.

Bioinformatics analysis. All ChIP data were analyzed and processed using a unified procedure to eliminate data processing bias. Reads were filtered with Trimmomatic (version 0.32) (29) and then mapped to the hg19 genome using Bowtie (version 2.2.4) (30). Subsequent to normalization to the indicated counts, tdf files were generated to visualize the genomic coverage using IGVtools (version 2.3.52) (31). Only one mapped read to each unique region of the genome that was $>175$ bp was kept and used in peak calling. Peak calling and motif analyses were then performed using MACS (version 1.4.2) (32) and HOMER (version 4.1) (33) with default parameters. Motifs with lengths of 8,10 and 12 bases were used for de novo motif analysis. A simple script was developed to calculate the normalized read density of the ChIP-seq data in any given region. Each given site was extended to $2 \mathrm{~kb}$ from the center of the vertex, and the 4-kb region was divided into 40 bins in units of $100 \mathrm{bp}$. Subsequently, the density of reads per bin (100 bp) was calculated. In order to allow for comparisons among multiple groups, the density of the reads was normalized to the total mapped reads to produce a signal in units of reads per 10 million mapped reads per $100 \mathrm{bp}$. Super-enhancers were defined and calculated as previously described, with slight modifications $(24,26)$.

The gene expression profile platforms included Affymetrix (http://www.affymetrix.com/site/mainPage.affx) and PrimeView (http://www.primeviewglobal.com/), and data processing was performed with $\mathrm{R}$ software (version 3.3.4) using the affy packages (34). The data on gene transcript platforms, including HuEx-1_0-st and HuGene-1_0-st, were analyzed using the oligo package (version 1.48.0, http://www. bioconductor.org/packages/release/bioc/html/oligo.html). The original data were normalized using the Robust Multi-array Average method $(35,36)$, whereas differentially regulated genes were identified using the limma package (37). A false discovery rate (FDR) of $<0.01$ was set as the screening standard. Gene set enrichment analysis (GSEA) of the core gene set regulated by JQ1 was also performed among the test set (38). Enrichment analysis was performed with Gene Ontology (GO) and the Encyclopedia of Genes Genomes (KEGG) pathway (https://david.ncifcrf.gov/).

Statistical analysis. One-way analysis of variance based on the function 'aov' in $\mathrm{R}$ software (version 3.4.5) was performed to analyze the overall differences Tukey and Student-Newman-Keuls tests based on functions of 'TurkeyHSD' and 'SNK.test' in package agricolae (version 1.3-1) in R software (version 3.4.5) were conducted to assess multiple comparisons between different groups. A statistically significant difference was indicated by $\mathrm{P}<0.05$.

\section{Results}

Most BRD4 sites bind to cell type-specific enhancer regions, while a small portion of significantly enriched sites bind to the promoter regions of all cell lines. In order to investigate the distribution of BRD4 genome-wide binding in multiple cell lines, the BRD4 sites from the six cell lines examined in the present study were merged into one collection. Two or more binding sites with overlapping positions ( $\geq 1 \mathrm{bp}$ ) on the genome were merged into one binding interval, and this interval was set as being shared by the cell line to which the binding site belonged. Thus, the binding sites of the six cell lines, including H2171 ( $\mathrm{n}=24,273)$, HUVEC-C $(\mathrm{n}=10,173)$, KOPT-K1 $(n=17,975)$, Ly1 $(n=18,696)$, MM.1S $(n=20,768)$ and U-87 $(n=29,216)$, were merged into 76,946 BRD4 binding intervals (Table SI). In theory, each final merged BRD4 binding intervals will be yes or no when considering whether or not it belongs to the binding site of BRD4 in the certain cell line of the six cell lines. The number of all combinations in all the six cell lines is theoretically $2^{6}-1$ except that the group S000000, which represents binding sites, does not belong to any of the six cell lines. Finally, the total number of binding sites for all cell lines was theoretically divided into 63 groups (calculated from $2^{6}-1$ ) according to the cell lines in which each BRD4 binding interval was located.

To evaluate the statistical significance of the number of binding sites in each group, an independent random sampling method was used to calculate a background number for each group. The BRD4 binding sites of the six cell lines were integrated into one collection (without merging between different binding) and the same number sites for each cell was randomly extracted from the collection as BRD4 binding sites in each corresponding cell line. Then, the six random extracted groups were merged into one collection. By repeating the aforementioned steps 1,000 times, a random background for the number of sites in each group was constructed. Assuming that the background had a normal distribution, the statistical 
Z-score value was calculated. According to the order of the number of binding sites in each group from large to small (for numbers <250), it was revealed that the number of BRD4 binding sites that were cell line-specific and only occurred in one cell line was the highest. A total of $96.68 \%$ of the BRD4 binding events were cell line-specific. As presented in Table I, the cell line-specific BRD4 binding sites in the U-87 and H2171 cell lines accounted for 29.67 and $27.72 \%$ of the total number of BRD4 binding sites, respectively, and represented 60.16 and $67.65 \%$ of the BRD4 binding sites in the respective cell lines. These results indicated that the genome-wide distribution of BRD4 binding sites differs greatly among the different cell lines. In addition, it was revealed that only 1,509 BRD4 (S111111) sites were shared by all cell lines; however, their significance was high when compared to the background distribution generated by the number of randomly sampling group of S111111 with significant z-score, indicating that BRD4 binding in all cell lines was not a random event (Table I). In order to study the BRD4 binding sites shared by multiple cell lines, BRD4 binding sites that co-existed in at least five of the cell lines were defined as a common BRD4 set.

To further analyze the distribution characteristics of BRD4 in the functional region and its interaction with various associated factors (10), a number of histone modifications and coactivators, namely H3K27Ac, H3K4me3, MED1, MYC, MAX and RNAPII, were integrated relative to the BRD4 function. The binding intervals of BRD4 that are presented in Table I were used as potential BRD4 binding sites in all cell lines, and their binding densities were then extracted. Thus, it was possible to quantitatively analyze the correlation between BRD4 and associated factors surrounding the potential BRD4 binding sites. Based on the correlation analysis of various factors, it was indicated that all factors could be divided into two categories. The first category is a cell line-independent class that mainly includes the histone modification $\mathrm{H} 3 \mathrm{~K} 4 \mathrm{me} 3$, the TFs MYC and MAX, and RNAPII (Fig. 1A). In general, transcription start sites and promoter regions of actively transcribed genes are marked by $\mathrm{H} 3 \mathrm{~K} 4 \mathrm{me} 3$, H3K27Ac and RNAPII (39), while MYC/MAX dimers have also been reported to be mainly incorporated in the promoter regions of genes (40). This observation suggests that several of the aforementioned factors may be associated with the BRD4 sites due to their significant enrichment in multiple cell lines and distribution in the gene promoter region. The second category is a cell line-specific class, in which each subset comprises BRD4, H3K27Ac and/or MED1, which indicates that the majority of the BRD4 sites in the genome bind by recognition of the acetyl group of H3K27Ac and are co-occupied by MED1. Active enhancers may be identified by enrichment of monomethylated H3K4 and H3K27Ac (41), and MED1 is a co-activated complex consisting of a linker enhancer and a promoter. Therefore, it may be concluded that the BRD4 binding site is mostly cell line-specific and is highly correlated with the enhancer region. In addition, according to the clustering distance, the distribution of BRD4 in the MM.1S, KOPT-K1 and Lyl cells was closer, and their common binding sites (S001110) were also significant ( $\mathrm{z}$-score $=271.45$ ); by contrast, U-87 was closer to the distribution in HUVEC-Cs, and the $\mathrm{z}$-score of their common binding site (S010001) was 162.
Table I. Number of each group in the overall union of BRD4 binding sites.

\begin{tabular}{|c|c|c|c|c|}
\hline Group & $\begin{array}{c}\text { Peak } \\
\text { numbers }\end{array}$ & $\begin{array}{c}\text { Percentage } \\
(\%)\end{array}$ & $\begin{array}{c}\text { bg } \\
\text { counts }\end{array}$ & zscore \\
\hline S000001 & 17,576 & 29.67 & 7,134 & $1,236.56$ \\
\hline S100000 & 16,420 & 27.72 & 5,590 & $1,364.17$ \\
\hline S000100 & 7,009 & 11.83 & 4,040 & 408.01 \\
\hline S000010 & 6,740 & 11.38 & 4,589 & 281.63 \\
\hline S001000 & 5,413 & 9.14 & 3,852 & 218.53 \\
\hline S010000 & 4,119 & 6.95 & 2,014 & 328.88 \\
\hline S010001 & 1,642 & 2.77 & 803 & 161.96 \\
\hline S111111 & 1,509 & 2.55 & 293 & 304.88 \\
\hline S001010 & 1,476 & 2.49 & 986 & 89.23 \\
\hline S001110 & 1,411 & 2.38 & 306 & 271.45 \\
\hline S001111 & 1,288 & 2.17 & 338 & 228.33 \\
\hline S000110 & 1,146 & 1.93 & 1,032 & 20.82 \\
\hline S101111 & 1,135 & 1.92 & 516 & 138.58 \\
\hline S001100 & 994 & 1.68 & 864 & 24.77 \\
\hline $\mathrm{S} 100001$ & 813 & 1.37 & 2,282 & -228.46 \\
\hline S011111 & 764 & 1.29 & 138 & 185.07 \\
\hline S000011 & 697 & 1.18 & 1,862 & -185.14 \\
\hline S001011 & 587 & 0.99 & 579 & 1.54 \\
\hline $\mathrm{S} 100010$ & 586 & 0.99 & 1,445 & -145.26 \\
\hline S101110 & 510 & 0.86 & 242 & 68.97 \\
\hline S100100 & 429 & 0.72 & 1,264 & -143.34 \\
\hline S000101 & 341 & 0.58 & 1,634 & -212.80 \\
\hline S101000 & 313 & 0.53 & 1,207 & -160.35 \\
\hline S001001 & 303 & 0.51 & 1,554 & -210.82 \\
\hline S101010 & 271 & 0.46 & 437 & -37.15 \\
\hline S101011 & 256 & 0.43 & 508 & -53.59 \\
\hline
\end{tabular}

BRD4, bromodomain containing 4 .

Next, a heatmap of BRD4 and its co-binding factors at each BRD4 binding interval was generated (Fig. 1B). The results revealed that, at cell line-specific BRD4 binding sites, only BRD4 and H3K27Ac of the corresponding cell line have binding signals with a low density for H3K4me3 and RNAPII. In the BRD4 binding sites shared by multiple cell lines, BRD4, H3K27Ac, H3K4me3 and RNAPII were all enriched among all the cell lines examined. The binding sites of each group were further mapped to RefSeq genes to study the gene distributions (Fig. 1C). It was observed that the cell line-specific BRD4 binding sites are located distant from the gene transcription initiation site, and are mainly distributed in the enhancer and intergenic regions. By contrast, the BRD4 binding sites shared by multiple cell lines are mainly distributed in promoter regions, which was consistent with the results presented in Fig. 1A. X-box binding protein 1 (XBP1) has been reported to be highly expressed in MM and to have an important role in the genesis of this tumor (42). The binding signals of BRD4, H3K27Ac and RNAPII around the XBP1 gene in the six cell lines are presented in Fig. 1D. In the XBP1 upstream enhancer region, only the MM.1S cell line had a 
A

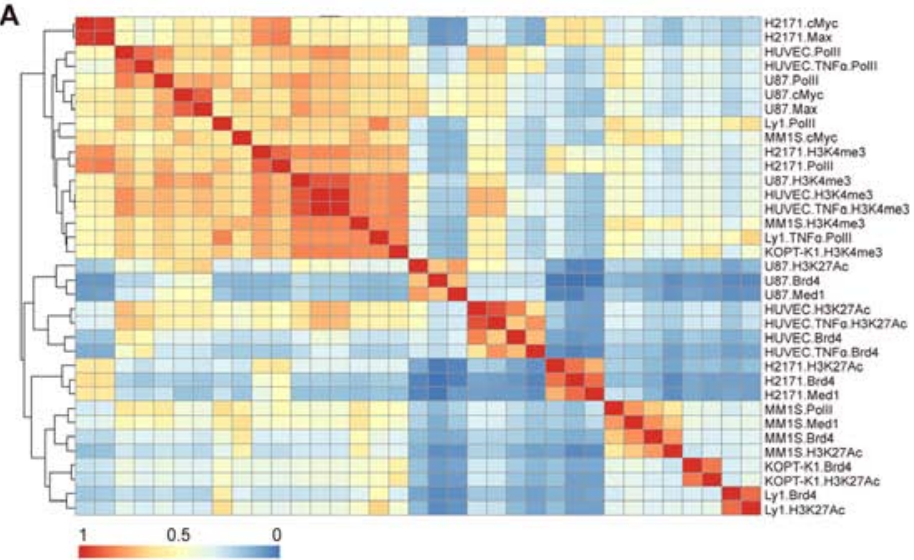

C

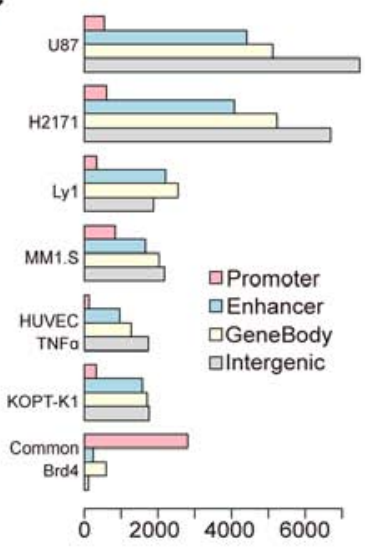

D

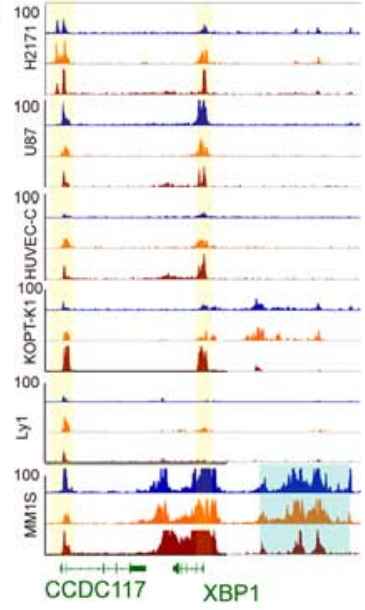

B

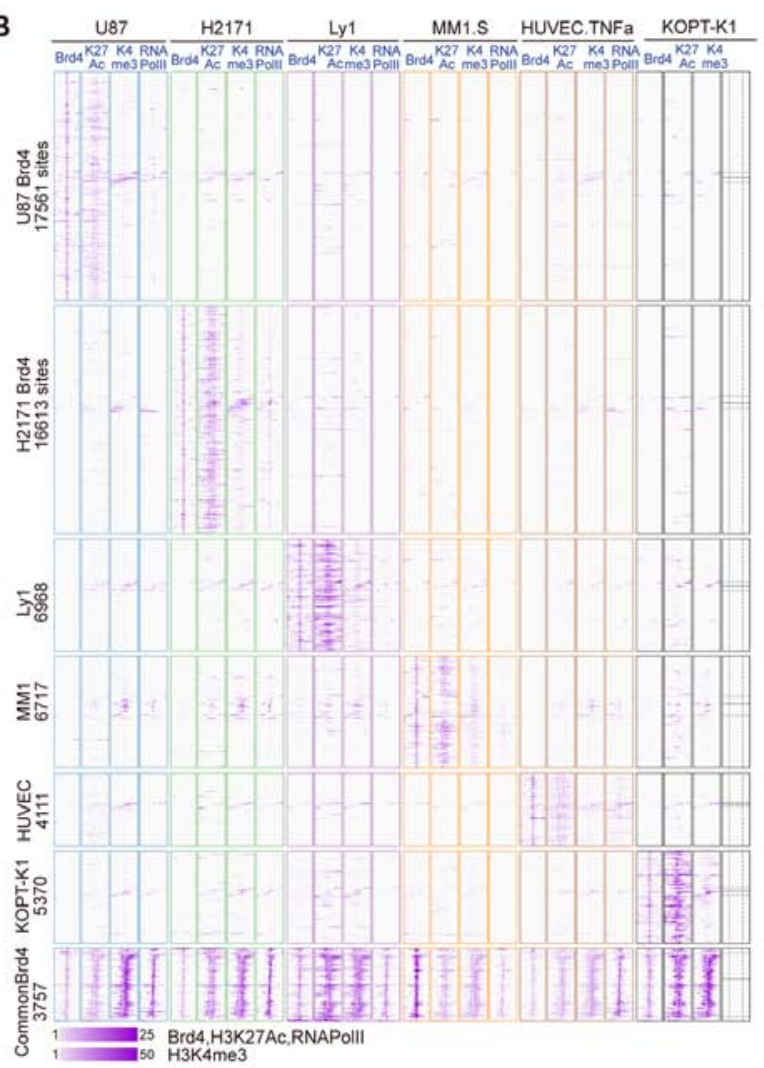

Figure 1. Genome-wide analysis of BRD4 binding characteristics. (A) Correlation analysis of BRD4 and its associated factors, including histone modifications (H3K27Ac and H3K4me3), co-activators (MED1 and MYC/MAX) and RNAPII, in six cell types in the merged BRD4 binding dataset. (B) Heatmap of ChIP-seq signals for BRD4, H3K27Ac, H3K4me3 and RNAPII centered on a $\pm 2.5 \mathrm{~kb}$ window of the BRD4 peak summit in the six cell types for seven groups of cell type-specific and cell type-common BRD4 binding sites. (C) Bar plots showing the genomic distribution associated with RefSeq genes of cell type-specific and cell type-common BRD4 binding sites. (D) Gene tracks of the ChIP-seq signals for BRD4, H3K27Ac and RNAPII at the XBP1 gene loci in all six cell types. The enhancer regions unique to the MM.1S cell line are highlighted with a blue shadow, while the promoter regions are highlighted with a yellow shadow.

high density of BRD4 and H3K27Ac, and a relatively low density of RNAPII. BRD4 and RNAPII were found to bind to the promoter regions of XBP1 and CCDC117 in multiple cell lines. Based on these results, the distribution of BRD4-binding sites in all the investigated cell lines was obtained. The majority of the BRD4 binding sites were found to only bind to specific cell lines and to co-localize with H3K27Ac and MED1 in enhancer regions. By contrast, a certain portion of BRD4 binding sites were observed to bind to all cell lines and to mainly co-bind with RNAPII, H3K4me3 and MYC/MAX in promoter regions.

Cell type-common BRD4 binding sites co-localize with oncogenic TFs, including E2FI and MYC, in promoter regions. To regulate transcription, BRD4 binds to a complex involved in transcription and interacts with a variety of TFs. To examine the interactions of cell type-specific and cell type-common BRD4 binding sites with specific TFs, a de novo motif analysis of these two categories of BRD4 sites was performed to obtain potential TFs that may bind in proximity to the two categories of BRD4 binding. It was observed that the cell line-specific TFs were significantly enriched at the cell line-specific BRD4 binding sites (Fig. 2A). The interferon-regulatory factor (IRF) family was specifically enriched in MM.1S cells, nuclear factor $\kappa \mathrm{B}(\mathrm{NF} \kappa \mathrm{B})-\mathrm{P} 65$ was specifically enriched in TNF- $\alpha$-activated HUVEC-Cs, and the CCAAT enhancer-binding protein family was specifically enriched in U-87 cells. In addition, the hematopoietic-specific TFs PU.1 and runt-related transcription factor 1 were enriched in the blood cancer cell lines MM.1S, KOPT-K1 and Ly1. Furthermore, the E2F, YY1 and ETS motifs were enriched in the common BRD4 binding sites shared among multiple cell lines.

Previous studies have demonstrated that E2F, YY1 and ETS1 are mainly involved in gene promoter regions and are associated with fundamental functions of cell lines and tumorigenesis (43). Furthermore, the BRD4 inhibitor JQ1 inhibits the proliferation of cancer cells mostly by depressing the expression of the proto-oncogenes MYC or N-MYC, which are also the major binding sites in the gene promoter region. Thus, it was hypothesized that the cell type-common BRD4 binding sites may co-localize with cancer-associated TFs, including E2F1 and MYC, in the promoter region. To validate this hypothesis, ChIP-seq data for E2F1 and MYC 
in multiple cell lines were collected. First, the co-localization of TF binding events with the cell type-common and cell type-specific BRD4 binding sites was assessed (Fig. 2B). The results indicated that the majority of the cell type-common BRD4 binding sites were bound by E2F1 and MYC, and that the co-localization ratio exceeded $80 \%$. The co-binding ratio of the TFs IRF4 and NFKB-P65 in the cell type-specific BRD4 binding sites was only $20 \%$. However, at the cell line-specific BRD4 binding sites, binding of E2F1 and MYC was low, with only $8 \%$ of the cell line-specific BRD4 contained in E2F1 binding sites in the Ly1 cell line.

To further illustrate the interaction of the cell type-common BRD4 binding sites with E2F1 and MYC, the average binding signal of each TF in the two groups of BRD4 binding sites was evaluated (Fig. 2C). The results revealed that the binding signals of E2F1 and MYC were significantly higher in the cell type-common BRD4 binding sites as compared with those in the cell line-specific sites. By contrast, P65 exhibited significantly higher binding at the cell line-specific BRD4 sites. Next, the distribution of sites co-bound by TFs and BRD4 in specific cell lines across the whole genome was assessed. The results indicated that $\mathrm{E} 2 \mathrm{~F} 1$ and $\mathrm{MYC}$ were mainly distributed in the promoter region, whereas IRF4 and P65 were mainly distributed in non-promoter regions (Fig. 2D). Finally, to further analyze the genome-wide interaction of BRD4 with E2F1 and MYC, the present study then focused on binding near transcription start sites (TSSs). As indicated in the binding profiles in Fig. 2E, BRD4 and E2F1 or MYC exhibited significant co-localization at TSSs with RNAPII binding, and it was suggested that BRD4 co-localized with E2F1 or MYC in the promoter regions in the whole genome, particularly at cell type-common BRD4 binding sites.

In summary, using motif scanning combined with analysis of TF binding profiles, it was indicated that cell type-common BRD4 binding sites co-localized with the oncogenic TFs E2F1 and MYC at promoter regions with subsequent expression of downstream target genes, resulting in the development of cancer. These findings also suggest a potential mechanism by which BRD4 inhibitors effectively inhibit the proliferation of various cancer types.

BRD4 super-enriched regions or genes in each cell line comprise cell type-specific and cell type-common gene sets. Recent studies have indicated that most BRD4 binding sites are super-enriched in certain regions that are termed as super-enhancers $(9,21)$, which actively regulate the expression of surrounding genes. However, the definition of super-enhancers in previous studies is limited to super-enrichment of co-activators, such as MED1, in non-promoter regions. Previous studies by our and other groups have indicated that BRD4 and MED1 are co-localized throughout the whole genome (6), and thus, BRD4 may also serve as a factor for defining super-activated regions. In addition, a continuous distribution and ultra-high enrichment of BRD4 was observed near gene promoters (Fig. 3B). Thus, the method used to scan for super-enhancers was partially modified using the binding signal of BRD4. Promoter regions containing a single BRD4 binding peak were removed to retain only those in which multiple BRD4 binding peaks were continuously distributed. This modified method was used to scan the super-enhancer regions of all six cell lines and to align them to the nearest RefSeq gene. Since the region scanned by this method contained partial promoters, these regions were defined as BRD4 super-loaded regions (or super-enriched regions) instead of super-enhancers. A total of 305-482 BRD4 super-loaded regions were scanned per cell line, as shown in Fig. 3A. The assignment of super-loaded regions to genes in each cell type is provided in the supplementary files (Tables SIII-SVIII for H2171, HUVEC-C, KOPT-K1, Ly1, MM.1S and U-87, respectively). Subsequently, the BRD4 super-enriched genes were analyzed, and it was revealed that the genes with the most abundant BRD4 binding sites were key TFs or genes associated with cell-specific functions in the corresponding cell lines. Namely, the gene IRF2 was identified in MM.1S cells, POU class 2 homeobox-associating factor 1 and BCL6 transcriptional repressor were identified in Ly1 cells, C-C motif chemokine ligand 2 and IL8 were identified in HUVEC-C cells, TCF7 was identified in KOPT-K1 cells, HMGA2 was identified in U-87 cells, and INSM transcriptional repressor 1 was identified in $\mathrm{H} 2171$ cells. These detected TFs have previously been reported to serve important roles in the corresponding cell lines (44-48). Notably, it was also observed that a small number of genes were super-loaded with BRD4 in multiple cell lines, particularly MYC and BCL2-related protein A1 (BCL2A1). A number of earlier studies have indicated that MYC and BCL2A1 are involved in infinite proliferation and cell cycle regulation during cancer development $(49,50)$. As presented in Fig. 3B, the MYC and BCL2A1 promoter regions in multiple cell lines contained clusters of super-enriched fragments of BRD4 and H3K27Ac, suggesting that MYC and BCL2A1 serve an indispensable role in the development of different types of cancer.

Cell type-common BRD4 super-loaded genes are sensitive to BRD4 inhibitors and are highly associated with tumorigenesis. The BRD4 super-loaded region contains most of the BRD4 binding signals and actively regulates a small group of functionally important genes. Therefore, identifying genes associated with the BRD4 super-loaded region was an important aim of subsequent analysis in the present study. A small number of genes were found to be associated with BRD4 super-enriched regions in multiple cell lines, suggesting their important functions in multiple cell types. In order to further determine the characteristics of these genes, the gene set was redefined. For BRD4 hyper-enriched regions obtained in a specific cell line, it was examined whether the region was bound by BRD4 in other cell lines. If $>2$ BRD 4 binding sites were detected in 5 or more cell lines in the given region, this region was defined as a BRD4 cell line-common super-enriched region. Otherwise, this region was defined as a BRD4 super-enriched region specific to a particular cell line. Subsequently, these two categories of BRD4 super-enriched regions were assigned to the nearest RefSeq gene, and the common region-associated genes comprised the core gene set (Table SIX). The cell line-common BRD4 super-enriched genes had evidently higher BRD4 signals in each cell line (Fig. 4A), whereas a strong BRD4 signal was only present in the particular cell line for the cell line-specific BRD4 super-enriched genes. These results indicated the regions of the two categories were reliably determined using the aforementioned method. 
A

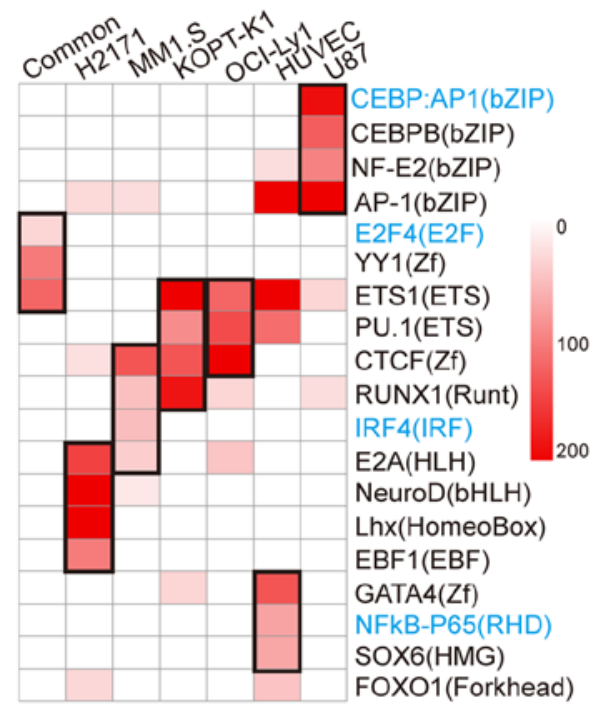

B

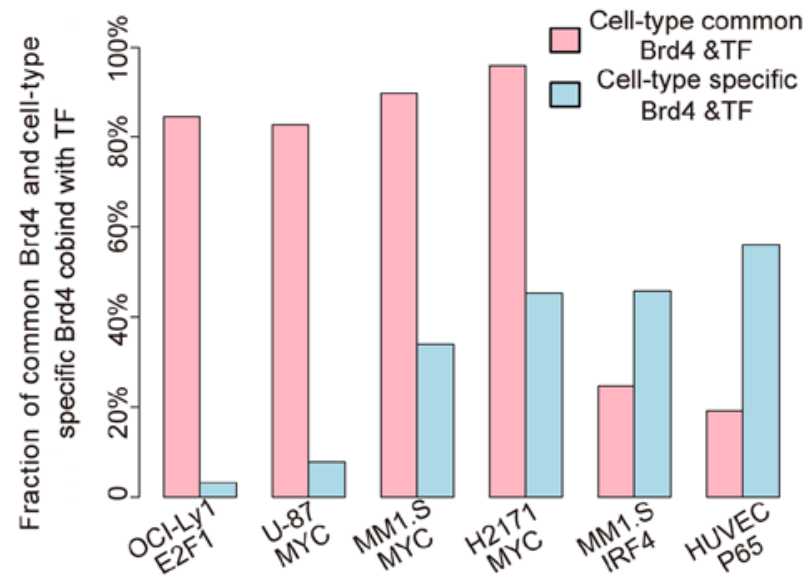

C

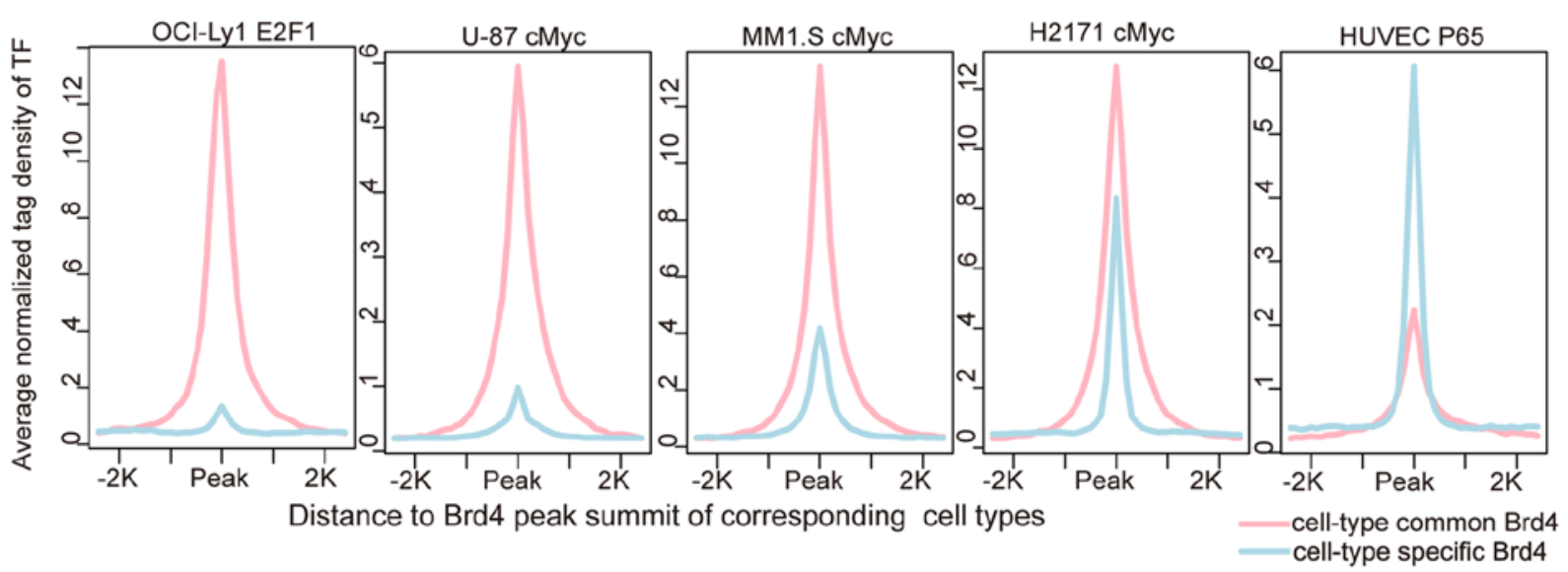

D

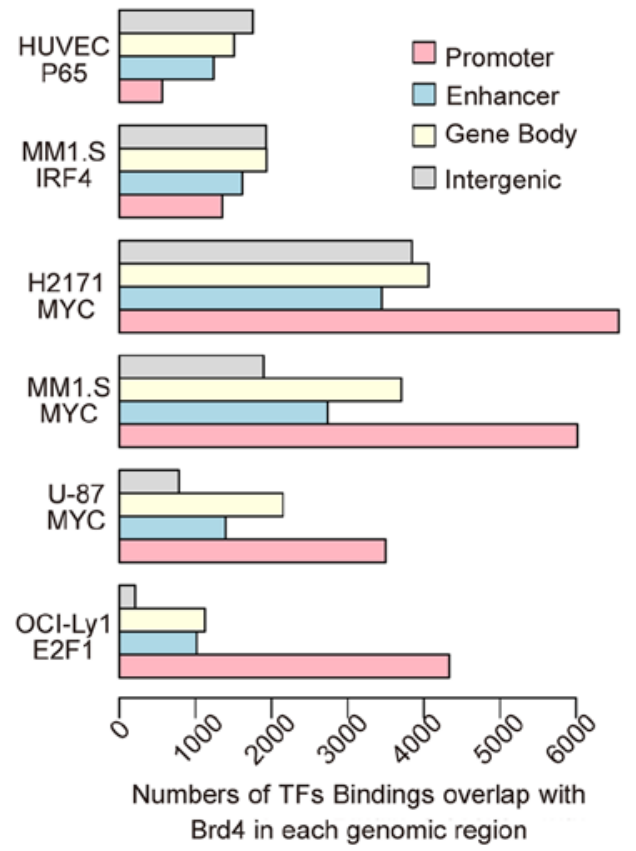

E

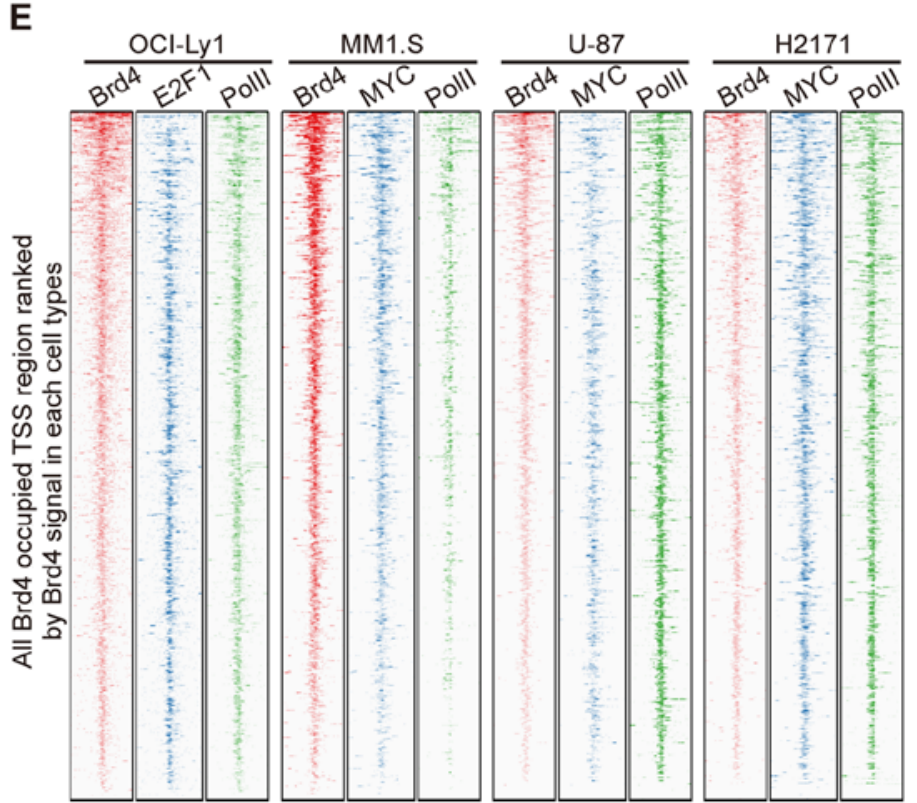

Figure 2. Interaction between BRD4 and TFs in a cell type-specific and cell type-common manner. (A) Motif enrichment of cell type-specific and cell

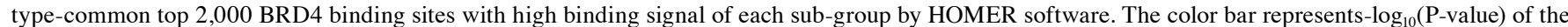
motif enrichment level. (B) Bar graphs displaying the fraction of cell type-common and cell type-specific BRD4 binding sites overlapped with TFs in the corresponding cell type, including E2F1 in Ly1 cells, MYC in U-87, MM.1S and H2171 cells, and IRF4 in MM.1S and NFkB-P65 in HUVEC-Cs. (C) Average density profiling of TF binding signals across cell type-common and cell type-specific BRD4 binding sites in each cell line. (D) Bar graphs displaying the genomic distribution associated with RefSeq genes of TF binding sites that overlapped with BRD4 in each cell line. (E) Heatmap of the chromatin immunoprecipitation sequencing signals for BRD4, RNAPII and TFs in each cell line at transcriptionally active promoters. Regions are centered on a $\pm 2.5 \mathrm{~kb}$ window around the transcription start sites and are ranked by RNAPII occupancy. 

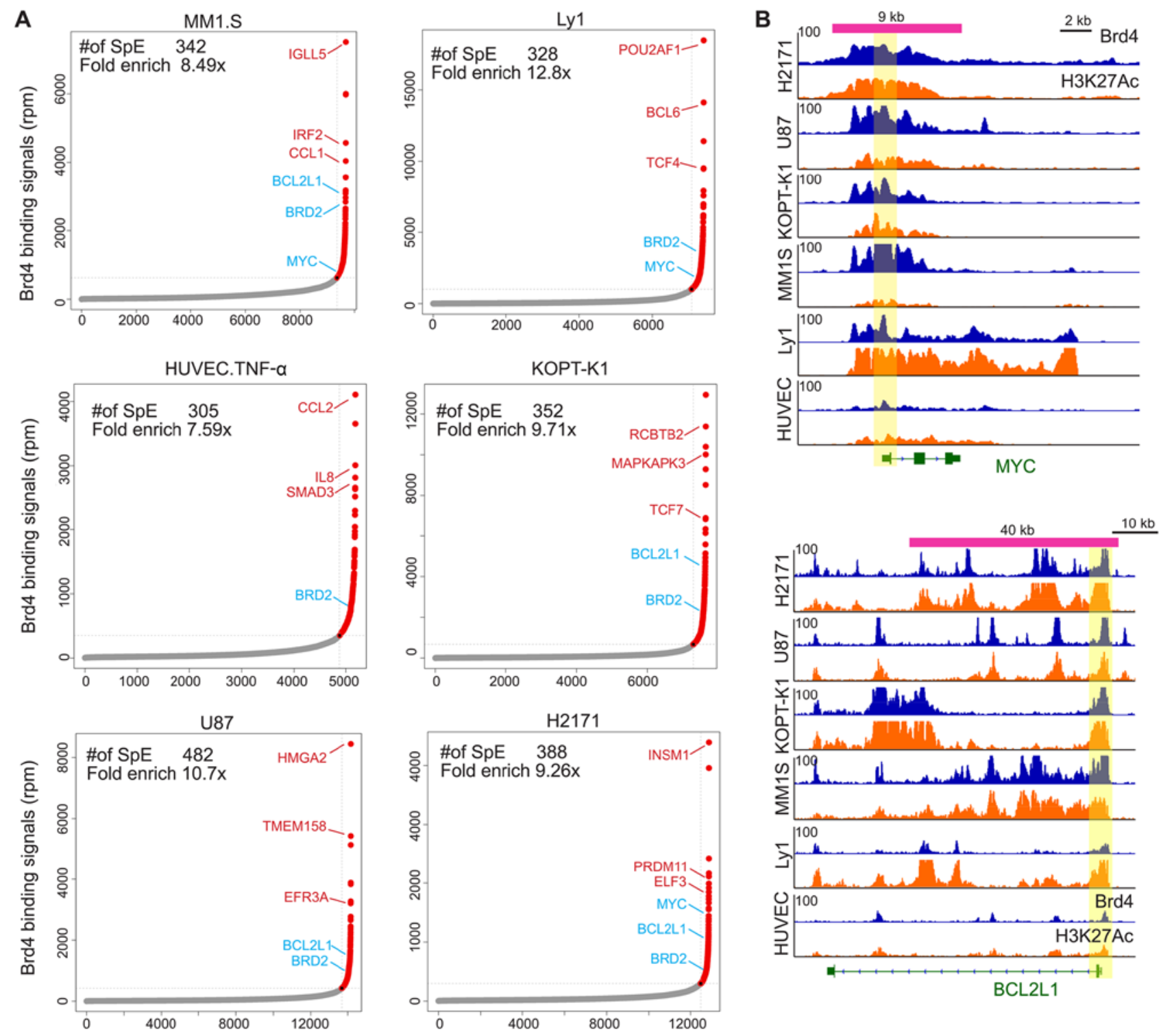

Figure 3. BRD4 super-loaded regions/genes determined in each cell line. (A) BRD4 ChIP-seq signal at BRD4 binding regions in each cell type. The regions are ranked by increasing BRD4 binding signals. BRD4 super-loaded regions are indicated in red, while other regions are indicated in grey. Cell type-specific genes are indicated in red and cell type-common genes in blue. (B) Gene traces of ChIP-seq signals for BRD4 and H3K27Ac at MYC and BCL2L1 gene loci in all six cell types. The BRD4 super-loaded regions are depicted with a red line, and the promoter regions are highlighted with a yellow shadow.

BRD4 regulates gene expression through a variety of mechanisms. To further study the expression of gene sets in each cell line, the expression profiles were collected, and the results were obtained by unified analysis (Fig. 4B and C). As indicated in the box plots in Fig. 4B, the expression of cell line-specific BRD4 super-enriched region-associated genes was significantly higher in the corresponding cell line as compared with that in the other cell lines. The box plots in Fig. 4C indicate that the cell line-common BRD4 super-enriched genes were significantly overexpressed in each cell line relative to all genes expressed in the genome. These results indicated that the BRD4 super enrichment-associated genes are actively transcribed in the genome, particularly the cell line-common BRD4 super-enriched genes, which are actively expressed in all cell lines. In addition, this potentially illustrates the importance of this gene set in cellular biological processes.
To further study the functions of genes linked to BRD4 super-enrichment, functional enrichment analysis among the different gene groups was performed. As presented in Fig. 4D, the Gene Ontology (GO) functional terms enriched by the cell line-specific gene groups were closely associated with the function of specific cell types. In the KOPT-K1 and Ly1 lymphoid cell lines, the GO categories that were specifically enriched by their genes are specific functions of lymphocytes, including immune regulation and activation, and the differentiation of B cells and T cells. HUVEC-Cs and U-87 cells are epithelial-derived cell lines, and their corresponding genes were significantly enriched in functions associated with cell adhesion, cell migration, angiogenesis, extracellular matrix and epithelial cells. Of note, the functional classes enriched by the cell line-common BRD4 super-enriched region-associated genes represented the basic functions during cellular biological processes, including gene transcription 
A

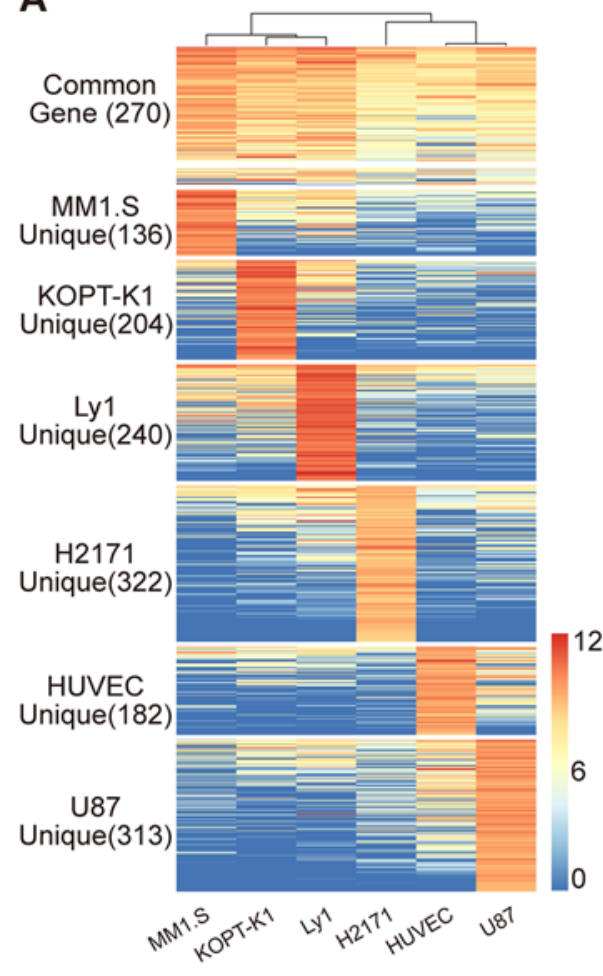

D

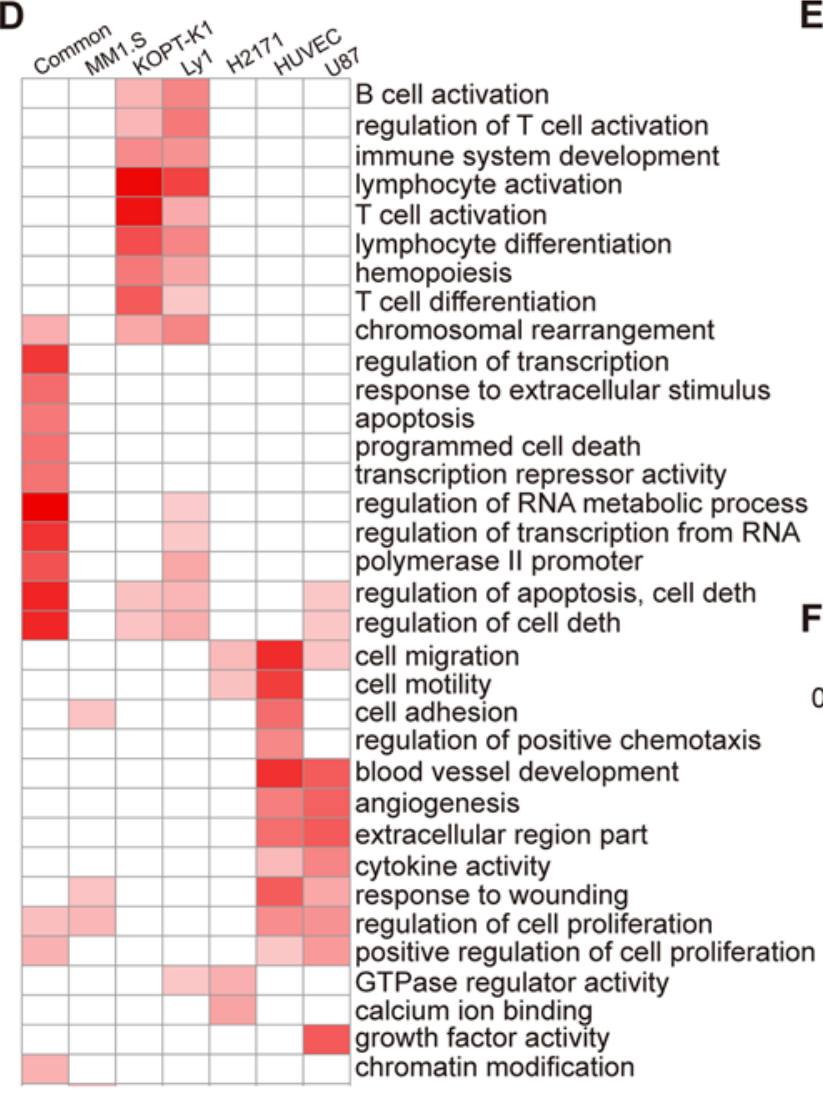

B
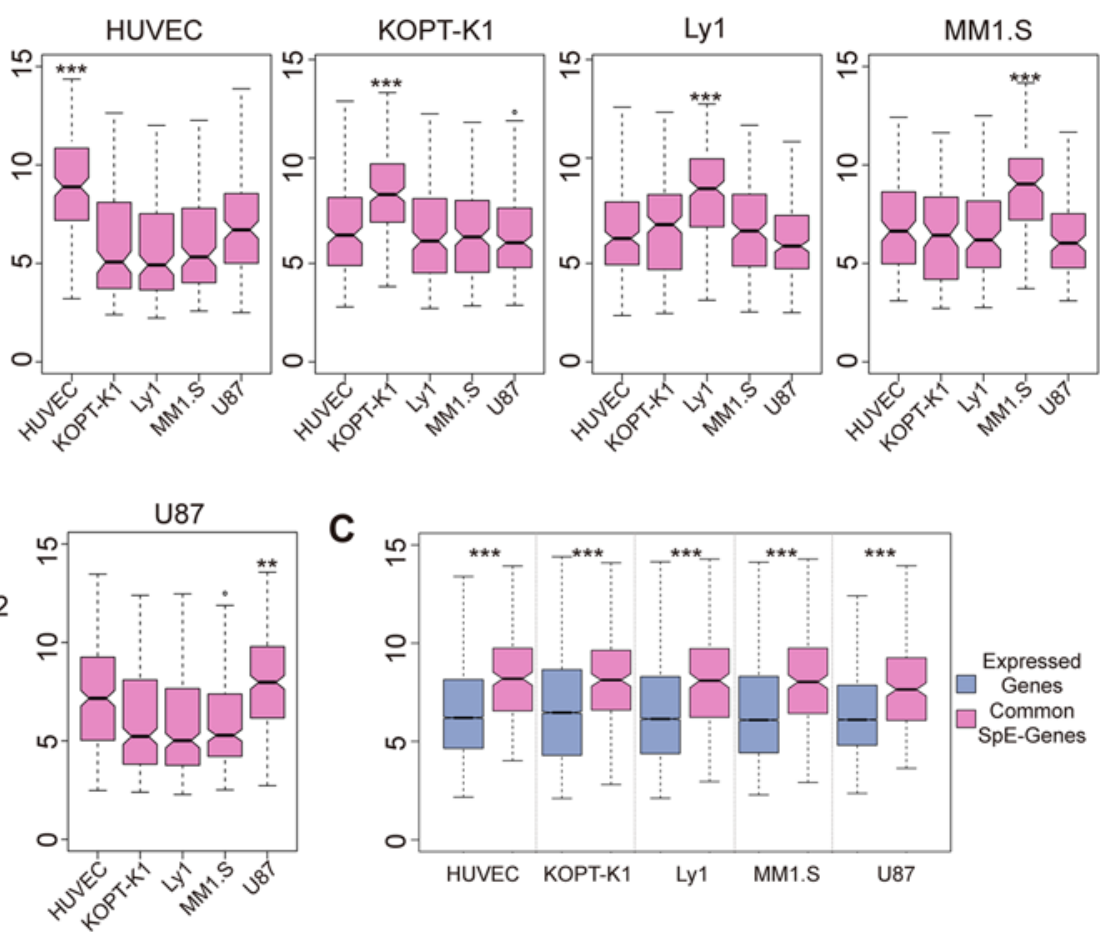

C

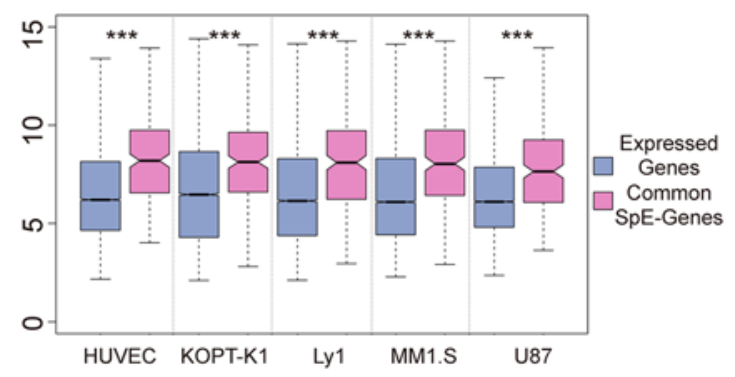

E

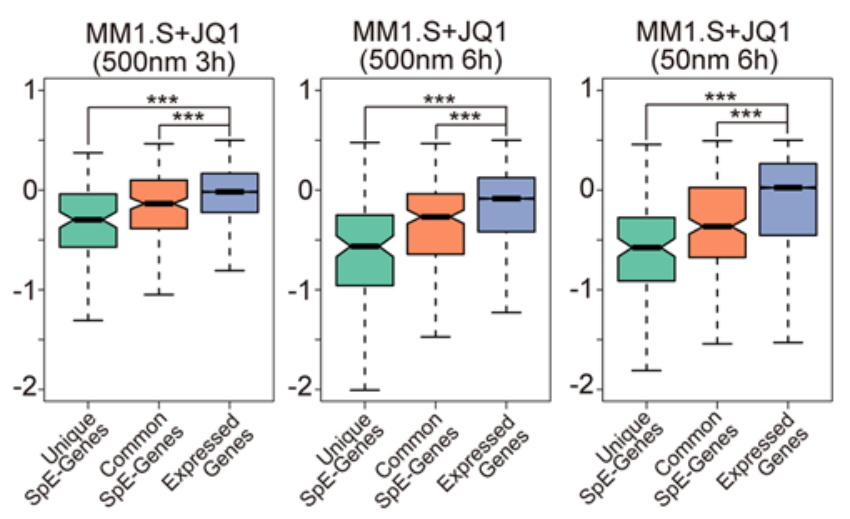

F

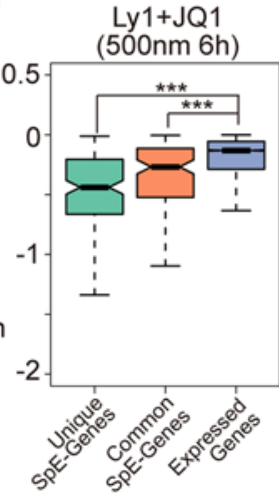

Ly1+JQ1 $(500 \mathrm{~nm} 12 \mathrm{~h})$

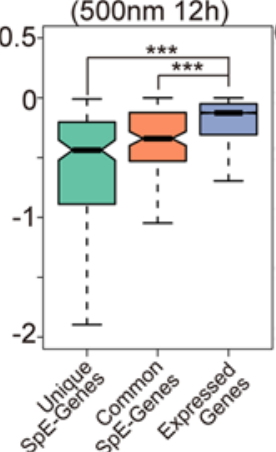

Ly1+JQ1 (500nm 24h)

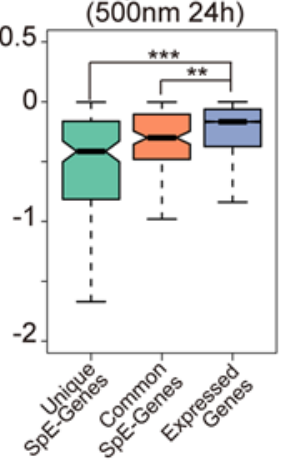

Figure 4. Functional annotation and effects of JQ1 on cell type-common and cell type-specific BRD4 super-loaded genes. (A) Heatmap of the log2-BRD4 ChIP-seq signal (rpm) at cell type-common and cell type-specific BRD4 super-loaded regions selected using a custom procedure. The numbers in the left panel indicate the BRD4 super-loaded target genes in each group. (B) Boxplots of cell type-specific BRD4 super-loaded target genes that are significantly actively transcribed in each cell line vs. other cell types. (C) Boxplot presenting cell type-common BRD4 super-loaded target genes that are significantly actively transcribed vs. all expressed genes in each cell type. (D) Heatmap displaying functional annotation enrichment of cell type-common and cell type-specific BRD4 super-loaded target genes generated by the DAVID website. The color bar represents $-\log _{10}(\mathrm{P}$-value $)$ of the functional enrichment level. Functional items with P-values of $<1 \times 10^{-3}$ in at least one cell type are included. (E) Boxplots of expression changes in MM.1S-specific and cell type-common BRD4 super-loaded target genes after JQ1 treatment at a concentration of 50 or $500 \mathrm{nM}$ and a duration of 3 or $6 \mathrm{~h}$ vs. all expressed genes in MM.1S cells. (F) Boxplots of expression changes among Ly1-specific, cell type-common BRD4 super-loaded target genes and all genes after treatment with $500 \mathrm{nM} \mathrm{JQ1} \mathrm{for} \mathrm{6,} 12$ and $24 \mathrm{~h}$. ${ }^{* *} \mathrm{P}<0.001$ and ${ }^{* * *} \mathrm{P}<0.0001$ (determined by a two-tailed t-test). 
regulation, RNA metabolism, apoptosis and necrosis, and cell proliferation.

The BRD4 inhibitor JQ1 is able to suppress BRD4 target genes, thereby effectively inhibiting tumor cell proliferation and promoting tumor cell apoptosis $(9,11)$. To test whether the BRD4 super-loaded target genes defined earlier in the study were sensitive to BRD4 inhibitors, the expression profiles of the MM.1S and Ly1 cell lines following JQ1 treatment were obtained to assess JQ1 inhibition of the aforementioned gene groups. The inhibitory effect of JQ1 on MM.1S-specific BRD4 super-loaded target genes was most evident at different concentrations and treatment times (Fig. 4E). In contrast to all the expressed genes, JQ1 also exerted a significant inhibitory effect on the cell-common BRD4 super-enriched genes. The results obtained with Ly1 cells were similar to those observed when the MM.1S cell line was subjected to JQ1 treatment (Fig. 4F), suggesting the selective inhibition of super-enriched BRD4 genes by JQ1 in specific and multiple cell lines.

Taken together, the cell line-specific and cell line-common BRD4 super-loaded regions were defined, and in-depth research on the expression, function and sensitivity of BRD4 hyper-accumulation-associated genes was performed. It was revealed that BRD4 hyper-accumulation-associated genes were actively transcribed in cells and that JQ1 had a significant inhibitory effect on their expression. The functional classes of cell line-specific gene groups were mainly enriched in specific functions of specific cell types, whereas the functional terms of the multi-cell shared gene groups were the basic functions of biological activities of cells, which were closely associated with the occurrence of cancer.

JQ1-targeted core gene group shared by multiple cell lines is significantly inhibited by JQ1 in different tumor types. The BRD4 inhibitor JQ1 has a good therapeutic effect on tumors of different origins $(11,13,14,25)$. It inhibits tumor proliferation and promotes apoptosis by inhibiting genes with BRD4 hyper-enrichment, thus affecting tumors with different sources $(6,23)$. The results of the present study suggested that super-enriched genes shared by multiple cells were significantly associated with cancer occurrence, and that JQ1 had an evident inhibitory effect on the expression of these genes (Fig. 4E). Therefore, it was hypothesized that JQ1 regulates a core gene group in different types of cancer cell to inhibit cancer. To examine whether any core gene group was regulated by JQ1, the 270 BRD4 super-enriched genes shared by multiple cell types were screened. As presented in Fig. 5A, genes that were significantly regulated by JQ1 were screened in at least two of the HUVEC-C, Lyl and MM.1S cell lines, as the JQ1 regulatory core gene group. The core regulatory genes of JQ1 contained a total of 67 genes (as indicated in Table SIX), among which MYC, BCL2, TCF4 and ETV6 were previously reported to have important roles in the development of various cancer types $(49,50)$. Notably, it was discovered that 8 genes were significantly upregulated by JQ1, particularly HEXIM P-TEFb subunit 1 (HEXIM1). HEXIM1 is a transcriptional elongation inhibitory factor that has a role opposite to that of BRD4 in the regulation of transcriptional elongation and may thus have a negative feedback effect on JQ1. Next, the current study examined whether the 67 JQ1 core regulatory genes were significantly inhibited by JQ1 in other cancer types. Expression data pre- and post-JQ1 treatment generated independently by different laboratories were collected as the test set, including for MM, BL, neuroblastoma and medulloblastoma cell lines. Similar to the GSEA results presented in Fig. 5B, the JQ1 core regulatory gene group was significantly inhibited by JQ1 in other cancer cell lines (FDR $<0.05)$. Subsequently, a functional enrichment analysis was performed on these 67 genes, indicating that their major functions were associated with transcriptional regulation and cancer development. A total of $45 \%$ of the genes in the core gene cluster (30/67) were TFs involved in the transcriptional regulation process, with 13 and 11 genes involved in apoptosis and cell proliferation, respectively, accounting for $36 \%$ of the total genes. In addition, 6 genes were involved in KEGG pathways, including MYC, BCL2L1, E2F3, transforming growth factor- $\beta$ receptor 2, TNF receptor-associated factor 1 and baculoviral IAP repeat containing 3 (Fig. 5C). Overall, the JQ1 core regulatory gene group was observed to serve an important role in a variety of cancer types, which provides a novel perspective on the role of BRD4 inhibitors in the treatment of cancer.

\section{Discussion}

BRD4 synergistically regulates the expression of downstream target genes by interacting with histone modifications, multiple co-activator complexes and TFs (51). The BRD4 inhibitor JQ1 competitively binds to BRD4 to inhibit its transcriptional activation effect and has been reported to have beneficial therapeutic effects in various cancer types $(11,13,15)$. In the present study, the genome-wide binding characteristics of BRD4 were systematically analyzed by integrating BRD4-associated data from multiple cell lines to unveil a general mechanism by which BRD4 inhibitors exert their anti-cancer effects. It was identified that cell line-specific BRD4 binding sites were co-localized with H3K27Ac and MED1 in enhancer regions, whereas cell line-common BRD4 binding sites co-localized with the proto-oncogene TFs MYC and E2F1 in gene promoter regions. Since genes distributed in BRD4 super-enriched regions have vital roles in cell biological processes, the present study also identified these genes in each cell line. The results revealed that the cell line-specific genes were associated with specific cell functions and cell identity. Conversely, the cell line-common genes were significantly enriched in fundamental cellular functions. Furthermore, these two categories were found to be sensitive to JQ1 treatment. In combination with the multiple expression profile data generated following JQ1 treatment, 67 core regulatory genes were finally screened from the BRD4 super-enriched gene set in various cancer cell lines that were suppressed by JQ1. The core regulatory genes mediated the general mechanism of BRD4 inhibitors, which provides valuable gene targets for further in-depth studies on transcriptional regulation and cancer treatment.

The ChIP-seq technique was also used to determine the characteristics of the distribution of BRD4 in the whole genome, providing a comprehensive perspective for the study of BRD4 in transcriptional regulation and tumor pathogenesis. It has previously been reported that BRD4 binds to almost all activated promoter regions across the genome, as well as most activated enhancer regions in a variety of cell types (52). The overall binding characteristics of BRD4 are similar to those 
A

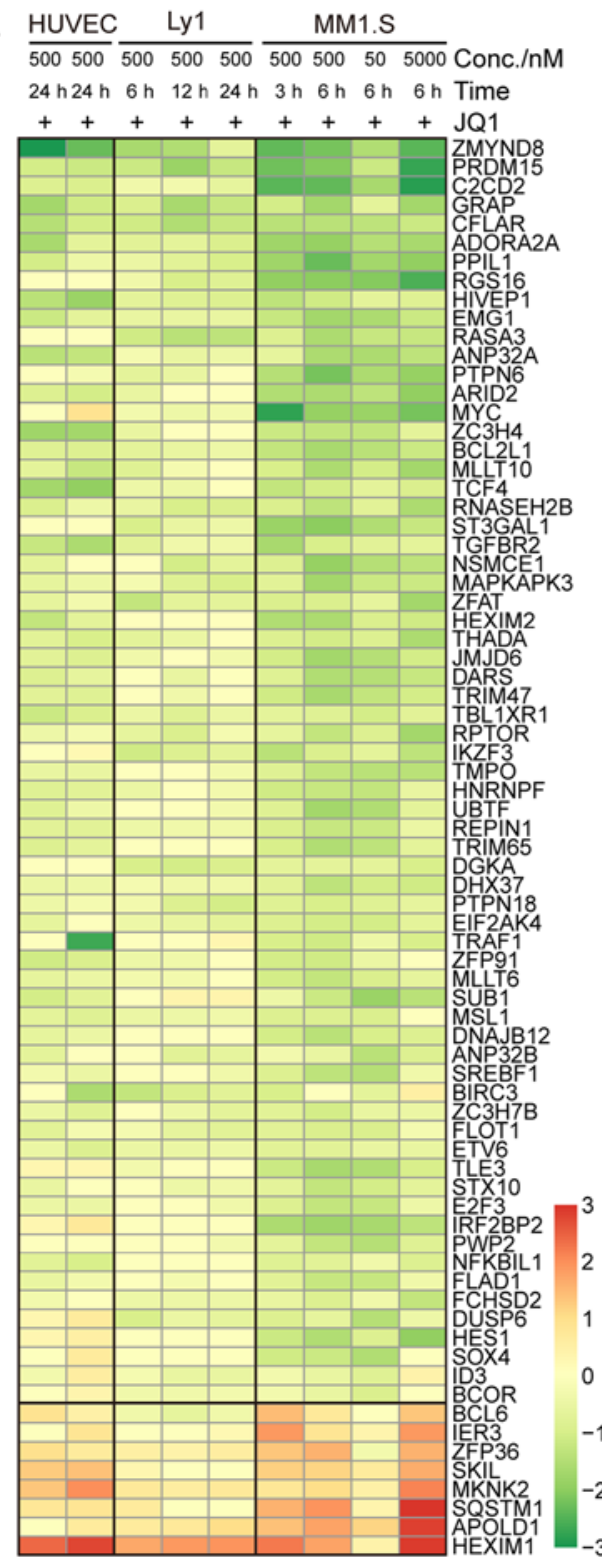

B
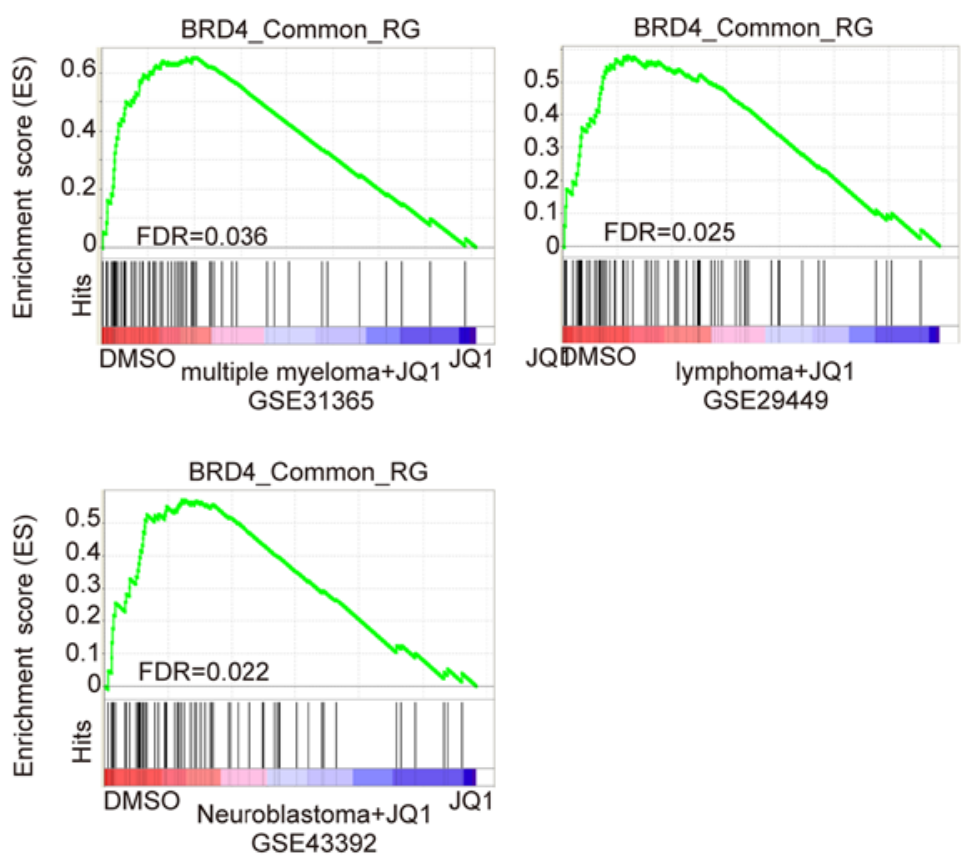

\begin{tabular}{l|r|l|l|l}
\hline Term & Count & PValue & Fold & FDR \\
\hline GO:0051252 regulation of RNA metabolic process & 26 & $8.68 \mathrm{E}-08$ & 3.13 & $1.36 \mathrm{E}-06$ \\
\hline GO:0045449 regulation of transcription & 30 & $5.00 \mathrm{E}-07$ & 2.52 & $7.85 \mathrm{E}-06$ \\
\hline $\begin{array}{c}\text { GO:0006357 regulation of transcription from RNA } \\
\text { polymerase II promoter }\end{array}$ & 15 & $3.15 \mathrm{E}-06$ & 4.50 & $4.95 \mathrm{E}-05$ \\
\hline $\begin{array}{c}\text { GO:0043066 negative regulation of apoptosis } \\
\text { GO:0060548 negative regulation of cell death }\end{array}$ & 10 & $2.71 \mathrm{E}-05$ & 6.16 & $4.26 \mathrm{E}-04$ \\
\hline GO:0042981 regulation of apoptosis & 10 & $3.10 \mathrm{E}-05$ & 6.06 & $4.86 \mathrm{E}-04$ \\
\hline GO:0001775 cell activation & 13 & $2.10 \mathrm{E}-04$ & 3.53 & $3.29 \mathrm{E}-03$ \\
\hline GO:0008284 positive regulation of cell proliferation & 8 & $2.94 \mathrm{E}-04$ & 6.08 & $4.61 \mathrm{E}-03$ \\
\hline GO:0042127 regulation of cell proliferation & 8 & $2.51 \mathrm{E}-03$ & 4.22 & $3.87 \mathrm{E}-02$ \\
\hline hsa05200:Pathways in cancer & 11 & $2.54 \mathrm{E}-03$ & 3.05 & $3.91 \mathrm{E}-02$ \\
\hline
\end{tabular}

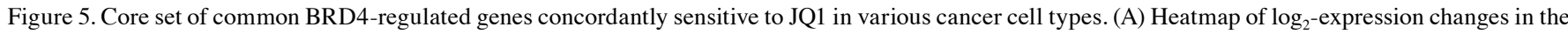
core set of common BRD4-regulated genes in HUVEC-C, Ly1 and MM.1S cells treated with JQ1 under various conditions. HUVEC-CH UVEC-C common BRD4 super-loaded genes that were significantly regulated [absolute $\log _{2}$ (fold change) $>0.75$ and FDR $<0.01$ ] by JQ1 in two of the three cell types were selected (B) Gene set enrichment analysis plot of the core set of the common BRD4-regulated gene set from gene expression profiling of different types of cancer cell lines treated with JQ1, including multiple myeloma (GSE31365), lymphoma (GSE29449) and neuroblastoma (GSE43392). (C) Functional enrichment analysis (performed with the DAVID online tool) of the core set of common BRD4-regulated genes. A count of $>5$ and P-value of $<0.01$ were considered to indicate statistical significance. BRD4, bromodomain containing 4; HUVEC-C, human umbilical vein endothelial cell; FDR, false discovery rate; TNF- $\alpha$, tumor necrosis factor $\alpha$; Conc., concentration; GO, Gene Ontology; hsa, Homo sapiens.

of histone acetylation modifications, including H3K9Ac and H3K27Ac, suggesting that it has the molecular characteristic of specifically recognizing histone acetylation. In the present study, ChIP-seq data were integrated from multiple cell lines and types of binding factors to reveal that BRD4 co-localized with a variety of factors in the promoter region, including H3K4me3, RNAPII and H3K27Ac, while it also bound to H3K27Ac and MED1 in the enhancer region. However, the genome-wide correlation analysis suggested that BRD4 was more closely associated with H3K27Ac and MED1 in all types of cell lines. In addition, most of the BRD4 binding sites exhibited a cell line-specific distribution and were localized in the enhancer region, whereas a small portion of the BRD4 binding sites coincided with the promoter region in multiple cell lines. These observations indicated that the binding distribution of BRD4 in the promoter region of different cell lines is more conservative, whereas the binding distribution in the enhancer region is more extensive and the cell line specificity is stronger.

BRD4 inhibitors were initially considered as effective MYC inhibitors. In MM.1S cells, which is an MM cell line that contains ectopic IgH/MYC, BRD4 binds in the distal IgH super-enhancer region and activates high MYC expression through long-term regulation (15). Recent studies have indicated that BRD4 synergistically regulates MYC expression by binding to the proximal promoter and distal enhancer regions 
of the MYC gene in various cancer cell lines with abnormal MYC expression $(14,16,53)$. In addition, BRD4 inhibitors inhibit tumorigenesis by reducing the expression of several key proto-oncogenes and their downstream target genes, such as MYC, BCL2 and E2F1 (17). In the present study, the results of the ChIP-seq binding site analysis suggested that BRD4 binding sites shared by multiple cell lines were co-localized with the key proto-oncogenes MYC and E2F1 in partially activated promoter regions. Gene expression profiling also revealed that genes downregulated by BRD4 inhibitors in multiple cell lines were significantly enriched in the regulatory sets of MYC and/or E2F1. These results indicated that BRD4 synergizes with MYC and E2F1 to activate its downstream target genes and promote cancer in a variety of cell lines, partially revealing the general mechanism of BRD4 inhibitors in various cell lines. However, overexpression of MYC or BCL2 in cancer cells treated with JQ1 only partially attenuated the anti-cancer effect of JQ1. This potentially suggests the diversity of the target and the complexity of the mechanisms of BRD4 inhibitors, which function not only through a key $\mathrm{TF}$ (such as MYC or E2F1), but also through a synergistic regulatory network that induces multiple TFs to exert an anti-cancer effect.

BRD4 is asymmetrically distributed in the genome and is called the super-enhancer region in a small portion $(<5 \%$ of the BRD4 binding sites) of regions that are enriched by $>50 \%$ of the BRD4 binding signal. The super-enhancer is localized in the transcription-activated enhancer region, which is distributed in the transcriptional co-activator MED1 and cell line key TF binding sites, and its associated genes are involved in cell identity and cell-specific functions (26). In the present study, it was revealed that the binding distribution of BRD4 and MED1 in the whole genome was consistent, and that BRD4 may be used to define the super-enhancer regions. In addition, a consecutive distribution of ultra-high BRD4 binding signals was observed around the promoter regions of numerous key genes. To completely scan the BRD4 super-loaded region, all enhancer regions and the promoter regions containing multiple BRD4 binding peaks were included. BRD4 super-loaded regions were obtained by scanning BRD4 signals. The present results indicated that the majority of the BRD4 cell line-common super-loaded regions contained gene promoter regions, and that the corresponding gene was actively expressed. These results suggested that BRD4 was super-enriched not only in a small part of the enhancer region, but also in the promoter region, and that the associated genes were implicated in fundamental cellular functions. Conversely, super-enhancer regions were mostly cell-specific binding regions, and their associated genes were involved in specific cell functions and cell identity decisions.

Previous studies have reported that BRD4 inhibitors have significant anti-cancer effects in inhibiting proliferation and promoting apoptosis in various cancer cell lines, particularly in hematological cancers (51). Furthermore, JQ1 has exhibited a broad and potent inhibitory effect in various human AML cell lines and patient samples. Subsequent mechanistic studies have suggested that BRD4 inhibitors significantly inhibit super-enhancer-associated genes (23). Therefore, the present study focused on the genes associated with BRD4 super-enrichment regions, which markedly narrowed the scope for subsequent research and refined its focus. Through multi-level screening, a core regulatory gene set around the BRD4 cell line-common super-loaded regions was obtained. Most of these genes were TFs closely linked to cancer, including the key proto-oncogene TFs MYC, BCL2L1 and $\mathrm{TCF} 4$. These findings suggest that the gene regulatory network of various types of cancer may contain a core gene group that is responsible for controlling fundamental cellular functions, including transcription, proliferation, apoptosis and the cell cycle. Finally, BRD4 inhibitors may exert their potent effect on various types of cancer by inhibiting this core gene set.

In conclusion, the present study found that BRD4 binds to enhancers with H3K27Ac and mediator in a cell-type specific manner and binds to the promoter region with oncogenic transcription factors MYC or E2F1 in a group of cell-type common binding sites. The genes with high BRD4 binding signal in cell-type specific super-enhancers and cell-type common super-enhancers were both sensitive to JQ1 treatment. The cell-type common ones across six cell types are functionally important for tumorigenesis, and the cell-type specific ones are functional enriched with cell identity. The present study obtained a core set of JQ1 regulated genes with cell-type common BRD4-super-loaded, which were significantly inhibited by JQ1 in many other cancer cell lines, and contributed to the cancer hallmarks. These results imply a common mechanism underlying therapeutic effects of JQ1, and provide a potential candidate for BRD4-mediated transcription regulation and BET-inhibitors-related cancer therapy.

\section{Acknowledgements}

Not applicable.

\section{Funding}

The present study was funded by the Zhejiang Provincial Department of Health Project (grant no. 2018KY515) and the Key Research Project of Traditional Chinese Medicine of Zhejiang Province of China (grant no. 2019ZZ015).

\section{Availability of data and materials}

The data of the present study are included in the manuscript and supplementary materials. The raw gene expression data are available from GEO (http://www.ncbi.nlm.nih.gov/geo/; accession nos. are provided in the supplementary materials), and the raw ChIP-Seq data are available on SRA (https://www. ncbi.nlm.nih.gov/sra/; accession nos. are provided in the supplementary materials).

\section{Authors' contributions}

The study was conceived and designed by GJ, CW and WD. WD, GJ and YL performed the experiments and analyzed the data, including sequence alignment. GJ and YL contributed the materials and analytical tools, as well as wrote and critically revised the content of the manuscript. $\mathrm{CW}$ also provided funding and guidance. All of the authors read and approved the final manuscript. 


\section{Ethics approval and consent to participate}

Not applicable.

\section{Patient consent for publication}

Not applicable.

\section{Competing interests}

The authors declare that they have no competing interests.

\section{References}

1. Dhalluin C, Carlson JE, Zeng L, He C, Aggarwal AK and Zhou MM: Structure and ligand of a histone acetyltransferase bromodomain. Nature 399: 491-496, 1999.

2. Belkina AC and Denis GV: BET domain co-regulators in obesity, inflammation and cancer. Nat Rev Cancer 12: 465-477, 2012.

3. Dey A, Chitsaz F, Abbasi A, Misteli T and Ozato K: The double bromodomain protein $\mathrm{Brd} 4$ binds to acetylated chromatin during interphase and mitosis. Proc Natl Acad Sci USA 100: 8758-8763, 2003 .

4. Jang MK, Mochizuki K, Zhou M, Jeong HS, Brady JN and Ozato $\mathrm{K}$ : The bromodomain protein $\mathrm{Brd} 4$ is a positive regulatory component of P-TEFb and stimulates RNA polymerase II-dependent transcription. Mol Cell 19: 523-534, 2005.

5. Jiang YW, Veschambre P, Erdjument-Bromage H, Tempst P, Conaway JW, Conaway RC and Kornberg RD: Mammalian mediator of transcriptional regulation and its possible role as an end-point of signal transduction pathways. Proc Natl Acad Sci USA 95: 8538-8543, 1998.

6. Donner AJ, Ebmeier CC, Taatjes DJ and Espinosa JM: CDK8 is a positive regulator of transcriptional elongation within the serum response network. Nat Struct Mol Biol 17: 194-201, 2010.

7. Patel MC, Debrosse M, Smith M, Dey A, Huynh W, Sarai N, Heightman TD, Tamura T and Ozato K: BRD4 coordinates recruitment of pause release Factor P-TEFb and the pausing complex NELF/DSIF to regulate transcription elongation of interferon-stimulated genes. Mol Cell Biol 33: 2497-2507, 2013.

8. Liu W, Ma Q, Wong K, Li W, Ohgi K, Zhang J, Aggarwal A and Rosenfeld MG: Brd4 and JMJD6-associated anti-pause enhancers in regulation of transcriptional pause release. Cell 155 $1581-1595,2013$

9. Rahman S, Sowa ME, Ottinger M, Smith JA, Shi Y, Harper JW and Howley PM: The Brd4 extraterminal domain confers transcription activation independent of pTEFb by recruiting multiple proteins, including NSD3. Mol Cell Biol 31: 2641-2652, 2011.

10. Yang Z, Yik JH, Chen R, He N, Jang MK, Ozato K and Zhou Q Recruitment of $\mathrm{P}-\mathrm{TEFb}$ for stimulation of transcriptional elongation by the bromodomain protein Brd4. Mol Cell 19: 535-545, 2005.

11. Picaud S: Selective inhibition of BET bromodomains. Nature 468: 1067-1073, 2010.

12. Nicodeme E, Jeffrey KL, Schaefer U, Beinke S, Dewell S, Chung CW, Chandwani R, Marazzi I, Wilson P, Coste H, et al: Suppression of inflammation by a synthetic histone mimic. Nature 468: 1119-1123, 2010

13. Dawson MA, Prinjha RK, Dittmann A, Giotopoulos G, Bantscheff M, Chan WI, Robson SC, Chung CW, Hopf C, Savitski MM, et al: Inhibition of BET recruitment to chromatin as an effective treatment for MLL-fusion leukaemia. Nature 478: 529-533, 2011.

14. Zuber J, Shi J, Wang E, Rappaport AR, Herrmann H, Sison EA, Magoon D, Qi J, Blatt K, Wunderlich M, et al: RNAi screen identifies Brd4 as a therapeutic target in acute myeloid leukaemia. Nature 478: 524-528, 2011

15. Delmore JE, Issa GC, Lemieux ME, Rahl PB, Shi J, Jacobs HM, Kastritis E, Gilpatrick T, Paranal RM, Qi J, et al: BET bromodomain inhibition as a therapeutic strategy to target c-Myc. Cell 146: 904-917, 2011.

16. Mertz JA, Conery AR, Bryant BM, Sandy P, Balasubramanian S, Mele DA, Bergeron L and Sims RJ III: Targeting MYC dependence in cancer by inhibiting BET bromodomains. Proc Natl Acad Sci USA 108: 16669-16674, 2011.
17. Chapuy B, McKeown MR, Lin CY, Monti S, Roemer MG, Qi J, Rahl PB, Sun HH, Yeda KT, Doench JG, et al: Discovery and characterization of super-enhancer-associated dependencies in diffuse large B cell lymphoma. Cancer Cell 24: 777-790, 2013.

18. Knoechel B, Roderick JE, Williamson KE, Zhu J, Lohr JG, Cotton MJ, Gillespie SM, Fernandez D, Ku M, Wang H, et al: An epigenetic mechanism of resistance to targeted therapy in $\mathrm{T}$ cell acute lymphoblastic leukemia. Nat Genet 46: 364-370, 2014.

19. Puissant A, Frumm SM, Alexe G, Bassil CF, Qi J, Chanthery YH, Nekritz EA, Zeid R, Gustafson WC, Greninger P, et al: Targeting MYCN in neuroblastoma by BET bromodomain inhibition. Cancer Discov 3: 308-323, 2013.

20. Bandopadhayay P, Bergthold G, Nguyen B, Schubert S, Gholamin S, Tang Y, Bolin S, Schumacher SE, Zeid R, Masoud S, et al: BET bromodomain inhibition of MYC-amplified medulloblastoma. Clin Cancer Res 20: 912-925, 2014.

21. Lockwood WW, Zejnullahu K, Bradner JE and Varmus H: Sensitivity of human lung adenocarcinoma cell lines to targeted inhibition of BET epigenetic signaling proteins. Proc Natl Acad Sci USA 109: 19408-19413, 2012.

22. Asangani IA, Dommeti VL, Wang X, Malik R, Cieslik M, Yang R, Escara-Wilke J, Wilder-Romans K, Dhanireddy S, Engelke C, et al: Therapeutic targeting of BET bromodomain proteins in castration-resistant prostate cancer. Nature 510: 278-282, 2014

23. Lovén J, Hoke HA, Lin CY, Lau A, Orlando DA, Vakoc CR, Bradner JE, Lee TI and Young RA: Selective inhibition of tumor oncogenes by disruption of super-enhancers. Cell 153: 320-334, 2013.

24. Whyte WA, Orlando DA, Hnisz D, Abraham BJ, Lin CY, Kagey MH, Rahl PB, Lee TI and Young RA: Master transcription factors and mediator establish super-enhancers at key cell identity genes. Cell 153: 307-319, 2013.

25. Di Micco R, Fontanals-Cirera B, Low V, Ntziachristos P, Yuen SK, Lovell CD, Dolgalev I, Yonekubo Y, Zhang G, Rusinova E, et al: Control of embryonic stem cell identity by BRD4-dependent transcriptional elongation of super-enhancer-associated pluripotency genes. Cell Rep 9: 234-247, 2014.

26. Hnisz D, Abraham BJ, Lee TI, Lau A, Saint-André V, Sigova AA, Hoke HA and Young RA: Super-enhancers in the control of cell identity and disease. Cell 155: 934-947, 2013.

27. Wu SY, Lee AY, Lai HT, Zhang $\mathrm{H}$ and Chiang CM: Phospho switch triggers Brd4 chromatin binding and activator recruitment for gene-specific targeting. Mol Cell 49: 843-857, 2013.

28. Brown JD, Lin CY, Duan Q, Griffin G, Federation A, Paranal RM, Bair S, Newton G, Lichtman A, Kung A, et al: NF- $\kappa$ B directs dynamic super enhancer formation in inflammation and atherogenesis. Mol Cell 56: 219-231, 2014

29. Bolger AM, Lohse M and Usadel B: Trimmomatic: A flexible trimmer for Illumina sequence data. Bioinformatics 30 : 2114-2120, 2014

30. Langmead B, Trapnell C, Pop M and Salzberg SL: Ultrafast and memory-efficient alignment of short DNA sequences to the human genome. Genome Biol 10: R25, 2009.

31. Thorvaldsdottir H, Robinson JT and Mesirov JP: Integrative Genomics Viewer (IGV): High-performance genomics data visualization and exploration. Brief Bioinform 14: 178-192, 2013.

32. Zhang Y, Liu T, Meyer CA, Eeckhoute J, Johnson DS, Bernstein BE, Nusbaum C, Myers RM, Brown M, Li W and Liu XS: Model-based analysis of ChIP-Seq (MACS). Genome Biol 9: R37, 2008.

33. Heinz S, Benner C, Spann N, Bertolino E, Lin YC, Laslo P, Cheng JX, Murre C, Singh H and Glass CK: Simple combinations of lineage-determining transcription factors prime-regulatory elements required for macrophage and B cell identities. Mol Cell 38: 576-589, 2010.

34. Gautier L, Cope L, Bolstad BM and Irizarry RA: affy-analysis of Affymetrix GeneChip data at the probe level. Bioinformatics 20: 307-315, 2004.

35. Irizarry RA, Bolstad BM, Collin F, Cope LM, Hobbs B and Speed TP: Summaries of Affymetrix GeneChip probe level data. Nucleic Acids Res 31: e15, 2003

36. Irizarry RA, Hobbs B, Collin F, Beazer-Barclay YD, Antonellis KJ, Scherf U and Speed TP: Exploration, normalization, and summaries of high density oligonucleotide array probe level data. Biostatistics 4: 249-264, 2003.

37. Gentleman RC, Carey VJ, Bates DM, Bolstad B, Dettling M, Dudoit S, Ellis B, Gautier L, Ge Y, Gentry J, et al: Bioconductor: Open software development for computational biology and bioinformatics. Genome Biol 5: R80, 2004. 
38. Subramanian A, Tamayo P, Mootha VK, Mukherjee S, Ebert BL, Gillette MA, Paulovich A, Pomeroy SL, Golub TR, Lander ES and Mesirov JP: Gene set enrichment analysis: A knowledge-based approach for interpreting genome-wide expression profiles. Proc Natl Acad Sci USA 102: 15545-15550, 2005.

39. Barski A, Cuddapah S, Cui K, Roh TY, Schones DE, Wang Z, Wei G, Chepelev I and Zhao K: High-resolution profiling of histone methylations in the human genome. Cell 129: 823-837, 2007.

40. Rahl PB and Young RA: MYC and transcription elongation. Cold Spring Harb Perspect Med 4: a020990, 2014.

41. Zhou VW, Goren A and Bernstein BE: Charting histone modifications and the functional organization of mammalian genomes. Nat Rev Genet 12: 7-18, 2011.

42. Claudio JO, Masih-Khan E, Tang H, Gonçalves J, Voralia M, Li ZH, Nadeem V, Cukerman E, Francisco-Pabalan O, Liew CC, et al: A molecular compendium of genes expressed in multiple myeloma. Blood 100: 2175-2186, 2002.

43. Whitfield TW, Wang J, Collins PJ, Partridge EC, Aldred SF, Trinklein ND, Myers RM and Weng Z: Functional analysis of transcription factor binding sites in human promoters. Genome Biol 13: R50, 2012.

44. Jelinek DF, Aagaard-Tillery KM, Arendt BK, Arora T, Tschumper RC and Westendorf JJ: Differential human multiple myeloma cell line responsiveness to interferon-alpha. Analysis of transcription factor activation and interleukin 6 receptor expression. J Clin Invest 99: 447-456, 1997.

45. Otsuki T, Yamada O, Sakaguchi H, Tomokuni A, Wada H Yawata Y and Ueki A: Human myeloma cell apoptosis induced by interferon-alpha. Br J Haematol 103: 518-529, 1998.

46. Mccoull W, Cheung T, Anderson E, Barton P, Burgess J, Byth K, Cao Q, Castaldi MP, Chen H, Chiarparin E, et al: Development of a novel B-cell lymphoma 6 (BCL6) PROTAC to provide insight into small molecule targeting of BCL6. ACS Chem Biol 13: 3131-3141, 2018
47. Rodriguez EF, Chowsilpa S and Maleki Z: insulinoma-associated protein 1 immunostain: A diagnostic tool for pulmonary small cell carcinoma in cytology. Acta Cytol 62: 333-338, 2018

48. Chen C, Breslin MB and Lan MS: Sonic hedgehog signaling pathway promotes INSM1 transcription factor in neuroendocrine lung cancer. Cell Signal 46: 83-91, 2018.

49. Gabay M, Li Y and Felsher DW: MYC activation is a hallmark of cancer initiation and maintenance. Cold Spring Harb Perspect Med 4: pii: a014241, 2014

50. Vogler M: BCL2A1: The underdog in the BCL2 family. Cell Death Differ 19: 67-74, 2012

51. Shi J and Vakoc CR: The mechanisms behind the therapeutic activity of BET bromodomain inhibition. Mol Cell 54: 728-736, 2014.

52. Anand P, Brown JD, Lin CY, Qi J, Zhang R, Artero PC, Alaiti MA, Bullard J, Alazem K, Margulies KB, et al: BET bromodomains mediate transcriptional pause release in heart failure. Cell 154: $569-582,2013$.

53. Lamoureux F, Baud'huin M, Rodriguez Calleja L, Jacques C, Berreur M, Rédini F, Lecanda F, Bradner JE, Heymann D and Ory B: Selective inhibition of BET bromodomain epigenetic signalling interferes with the bone-associated tumour vicious cycle. Nat Commun 5: 3511, 2014.

(7) $\ominus$ This work is licensed under a Creative Commons Attribution-NonCommercial-NoDerivatives 4.0 International (CC BY-NC-ND 4.0) License. 